\section{Taking a closer look at IBD}

\author{
Laurence J Egan, ${ }^{1}$ William Sandborn ${ }^{2}$
}

Healthcare professionals caring for patients with inflammatory bowel diseases (IBDs) in 2013 are equipped with significantly better knowledge about the disease, its variations, course and complications than in years gone by. Treatment options are improving, and perhaps more importantly, our knowledge of when and how to use existing treatments is becoming sharper. For these reasons, we have decided to publish a special issue of Gut in 2013 focusing on IBD. In this special edition, we have invited leading experts to review the current state of knowledge on six important aspects of IBD.

Perhaps the greatest recent scientific advances, enabled by high-throughput sequencing and other technologies, ${ }^{1}$ have been a deeper understanding of the genetic factors that are linked to IBD susceptibility in conjunction with alterations in the intestinal microbiome in patients. ${ }^{2} 3$ While much remains to be done in understanding the interactions between the genome and the microbiome, this avenue of investigation holds promise for major advances in the near future. The critical importance of genome-microbiome interactions lies in the potential to modulate the microbiome through dietary or other therapeutic interventions ${ }^{4}$ in specific patient populations. Such a development will open up a whole new chapter in our approach to the classification and management of IBD.

We also have the potential to learn a lot about the pathogenesis of intestinal inflammation from the study of monogenic diseases, which manifest with IBD-like clinical features. Categorisation of those disorders reveals groups of diseases characterised by defects in intestinal epithelial barrier function, immune deficiencies and disorders characterised by defects in $\mathrm{T}$ and $\mathrm{B}$ lymphocyte activity. Recent years have also seen very significant advances in our understanding of the pathogenesis of intestinal inflammation at the level of dysregulated immune cell function in the inflamed intestine.

Importantly, greater understanding of intestinal immunoregulation provides

\footnotetext{
'Discipline of Pharmacology and Therapeutics, NUI Galway, Galway, UK; ${ }^{2}$ Division of Gastroenterology, University of California San Diego, La Jolla, California, USA

Correspondence to Professor L J Egan, Discipline of Pharmacology and Therapeutics, NUI Galway, University Road, Galway, UK; Laurence.egan@nuigalway.ie
}

opportunities for productive research interfaces with many recently discovered IBD risk alleles, most notably those discovered in autophagy genes. ${ }^{6}$

While the results of clinical trials have provided best available evidence regarding the use of specific therapeutic interventions in groups of patients, there remains the unmet need to try to optimise therapies in day-to-day clinical practice. ${ }^{7}$ This knowledge gap has led to very significant efforts in developing better disease stratification tools than currently available. In the clinical management of IBD patients, a major current trend centres on developing approaches to individualised or personalised IBD management practices, where treatments are selected on objective grounds for specific patients. Our knowledge of the advantages and limitations of both blood-based and stool-based biomarkers, ${ }^{8}$ as well as imaging and endoscopic approaches has been significantly enhanced in recent years. ${ }^{9}$ Using combinations of these approaches, it will become possible to more precisely predict disease course in individual patients and tailor therapy accordingly.

In the clinical use of biological therapies for IBD, practising gastroenterologists are all too aware of the problems posed by primary non-response and secondary loss of response to anti-TNF- $\alpha$ monoclonal antibodies. ${ }^{10}$ Increasingly, there is availability of assays to measure blood levels of those drugs and antibodies to the drugs, an indicator of their immunogenicity. Significant clinical correlations have been established between higher trough drug levels and the absence of anti-drug antibodies, with better clinical outcomes. ${ }^{11}$ The results of ongoing clinical trials, which individualise dosing based on those parameters are eagerly awaited. The pipeline of new drugs for the treatment of IBD at this time is quite encouraging. Several new monoclonal antibodies targeting new pathways and mechanisms in the pathogenesis of intestinal inflammation, as well as some small molecule therapeutics that interfere with pro-inflammatory intracellular signalling pathways have shown promising recent results. Anti-adhesion antibodies with gut-specific activity also look set for introduction into the clinic in the near future. ${ }^{12} 13$ Increasingly, physicians caring for IBD patients will be able to select from a variety of new and highly effective drugs. A key challenge for the future will be to establish the relative effectiveness and risks of the available agents.

In summary, we have moved a long way from early discoveries on human leucocyte antigen-based risk alleles for IBD and from the rather blunt use of corticosteroids and 5 -aminosalicylic acid-based therapies for the treatment of IBD. The increasing prevalence of these diseases, coupled with an active and vibrant research agenda in recent decades, has allowed us to take a closer look at IBD. Most importantly, the real beneficiaries of this endeavour are patients.

Competing interests None.

Provenance and peer review Not commissioned; internally peer reviewed.

To cite Egan LJ, Sandborn W. Gut 2014;63:e1.

Received 6 June 2013

Accepted 6 June 2013

Gut 2014;63:e1. doi:10.1136/gutjnl-2013-305424

\section{REFERENCES}

1 Weinstock GM. Genomic approaches to studying the human microbiota. Nature 2012;489:250-6.

2 Lepage $P$, Leclerc MC, Joossens $M$, et al. A metagenomic insight into our gut's microbiome. Gut 2013;62:146-58.

3 Arthur JC, Perez-Chanona E, Muhlbauer $\mathrm{M}$, et al. Intestinal inflammation targets cancer-inducing activity of the microbiota. Science 2012;338:120-3.

4 Van den Abbeele P, Verstraete W, El Aidy S, et al. Prebiotics, faecal transplants and microbial network units to stimulate biodiversity of the human gut microbiome. Microb Biotechnol 2013;6:335-40.

5 Vigsnaes LK, van den Abbeele P, Sulek K, et al. Microbiotas from UC patients display altered metabolism and reduced ability of $L A B$ to colonize mucus. Sci Rep 2013;3:1110.

6 Parkes M, Barrett JC, Prescott NJ, et al. Sequence variants in the autophagy gene IRGM and multiple other replicating loci contribute to Crohn's disease susceptibility. Nat Genet 2007;39:830-2.

7 Travis S, Feagan BG, Rutgeerts $P$, et al. The future of inflammatory bowel disease management: combining progress in trial design with advances in targeted therapy. J Crohns Colitis 2012;6(Suppl 2):S250-9.

8 De Vos M, Dewit O, D'Haens G, et al. Fast and sharp decrease in calprotectin predicts remission by infliximab in anti-TNF naive patients with ulcerative colitis. J Crohns Colitis 2012;6:557-62.

9 Schoepfer AM, Beglinger C, Straumann A, et al. Fecal calprotectin correlates more closely with the Simple Endoscopic Score for Crohn's disease (SES-CD) than CRP, blood leukocytes, and the CDAI. Am J Gastroenterol 2010;105:162-9.

10 Sandborn WJ, Hanauer S, Loftus EV Jr, et al. An open-label study of the human anti-TNF monoclonal antibody adalimumab in subjects with prior loss of response or intolerance to infliximab for Crohn's disease. Am J Gastroenterol 2004;99:1984-9.

11 Ordas I, Feagan BG, Sandborn WJ. Drug monitoring of TNF antagonists in inflammatory bowel disease. Clin Gastroenterol Hepatol 2012;10:1079-87.

12 Sandborn WJ, Feagan BG, Rutgeerts $P$, et al. Vedolizumab as induction and maintenance therapy for Crohn's disease. N Engl J Med 2013 (In Press).

13 Feagan BG, Rutgeerts $P$, Sands BE, et al. Vedolizumab as induction and maintenance therapy for ulcerative colitis. N Engl J Med 2013 (In Press). 